\title{
" Tintin au Congo » devant les prétoires : Que peut signifier l'interdiction d'un héros de bande-dessinée belge ?
}

\begin{abstract}
La bande dessinée "Tintin au Congo " a soulevé ces derniers temps de nombreuses critiques. Éditée en 1931 et rééditée en 1946, certains passages

L'auteur : Vincent VESPA est titulaire d'un bachelier en droit subissent aujourd'hui le courroux de personnes jugeant l'ouvrage raciste et xénophobe. Dès lors, on peut s'interroger sur les significations d'une interdiction de cette bande-dessinée en Belgique ? Tel est l'objectif du présent article. Après avoir précisé les faits ayant remis l'ouvrage au cœur de l'actualité belge et internationale, les enjeux politiques d'une telle interdiction sont exposés. La liberté d'expression, la concurrence mémorielle - et son pendant, l'oubli -, ainsi que la reconnaissance des torts commis au "Congo Belge " sont successivement passés en revue. Ensuite, trois enjeux mémoriels liés à cette interdiction sont présentés : la négation de la représentation coloniale, la position du gouvernement congolais et les effets d'une jurisprudence interdisant une telle bande-dessinée. Enfin, en précisant l'époque à laquelle a été conçu " Tintin au Congo ", la conclusion revient sur les idées paternalistes des années 1930 tout en insistant sur l'intérêt d'un tel ouvrage, reflétant un imaginaire colonial et pouvant constituer un outil pédagogique pour les jeunes générations.
\end{abstract}

\section{Introduction}

Avec "Les Aventures de Tintin ", Hergé nous a plongés, dès notre plus tendre enfance, dans l'histoire d'un reporter qui fait le tour du monde et y résout une série d'énigmes. Sa deuxième œuvre aujourd'hui semble être contestée. En effet, un spectre plane sur "Tintin au Congo »... Celui du colonialisme des années 1930 et de ses idées paternalistes!

Cette bande dessinée, éditée en 1931 et rééditée en 1946 a aujourd'hui fait grincer les dents de Monsieur Bienvenu Mbutu Mondondo qui trouve cette dernière "raciste et xénophobe "' et pré-

"BeLGA, "Affaire Tintin au Congo: M. Mondondo débouté ", La Libre Belgique, 10 février 2012, disponible à l'adresse suivante : http://www.lalibre.be, (consultée ele 15 novembre 2012). 
tend qu'elle constitue " une insulte pour tous les Congolais " ${ }^{2}$. II a décidé de porter ce problème en justice et, dans ses conclusions, explique au juge les motifs qui le poussent à dire que la bande dessinée est raciste et xénophobe :

«[...] représentation graphique caricaturale des personnages africains, utilisation du terme 'nègre' dans la première édition de la bande dessinée, mauvaise maîtrise du français par les personnages africains, mise en scène de l'idée selon laquelle l'Africain se complait dans l'oisiveté, qu'il est naturellement paresseux (référence à l'épisode de la collision entre la voiture de Tintin et d'un train), paternalisme de Tintin qui pousse l'infantilisation des personnages africains à l'extrême (personnages africains qui se prosternent devant Tintin, épisode du chapeau coupé en deux) $n^{3}$.

Il est intéressant de se demander dans notre société diversifiée quels sont les problèmes que l'on peut rencontrer avec un ouvrage de cette époque. Que peut signifier l'interdiction de "Tintin au Congo" en Belgique? Nous allons essayer de répondre à cette question dans cet article qui se divisera en quatre parties.

La première consistera à expliquer quels sont les faits qui ont remis l'ouvrage au cœur de l'actualité belge mais aussi internationale. La deuxième traitera des enjeux politiques d'une telle interdiction. La troisième développera les enjeux mémoriels sur le sujet et sera suivie d'une conclusion.

\section{Faits remettant l'ouvrage aul courr de l'actualité}

\section{Les faits internationaux}

Plusieurs faits ont placé "Tintin au Congo » au devant de la scène internationale. Deux faits importants ont amené une réflexion sur le caractère raciste ou non de la bande-dessinée.

D'une part, en 2007, la commission britannique pour l'égalité des races (CRE) a demandé le retrait de la bande dessinée de toutes les librairies du Royaume-Uni après la plainte d'un citoyen. La chaîne Borders, en réponse à cette demande, a décidé de la placer dans les rayons adultes. II faut savoir que la vente de "Tintin au Congo" au Royaume-Uni se fait avec un bandeau rouge ${ }^{4}$ signalant que l'ouvrage

2. BeLGA, "Affaire Tintin au Congo: M. Mondondo débouté ", op. cit. 3. Civ. Bruxelles, 10 février 2012, n 10/4530/A \& 10/5711/A, 2012, p. 12. 4. DUPLAT Guy, «Tintin? Shocking! », La Libre Belgique, 13 juillet 2007, disponible à l'adresse suivante : http:// www.lalibre.be, (consultée le 16 novembre 2012). 
est destiné " aux collectionneurs » et véhicule " certains stéréotypes de l'époque qui peuvent choquer les lecteurs d'aujourd'hui »"

D'autre part, nous souhaitons mettre en avant le retrait de cette bande dessinée de la bibliothèque d'Arboga en Suède " en raison de son contenu raciste ". La bibliothèque d'Askersund quant à elle propose l'emprunt de l'ouvrage à des adultes, et à des enseignants ${ }^{6}$.

\section{Les faits belges}

En Belgique, une affaire judiciaire va être portée par Bienvenu Mbutu Mondondo, ressortissant congolais, accompagné par le Conseil Représentatif des Associations Noires (CRAN). Il faudra attendre pas moins de 5 ans pour que la justice belge donne son verdict?

Le 27 avril 2007, Monsieur Mbutu Mondondo porte plainte au pénal contre la SA Moulinsart et les éditions Casterman (deux affaires distinctes). Il estime que la bande dessinée porte atteinte à sa dignité d'" homme noir ". L'affaire "Tintin au Congo " débute.

Le 13 avril et le 3 mai 2010, il lance une citation en référé au Tribunal de première instance de Bruxelles sur base de la loi du 30 juillet 1981 tendant à réprimer certains actes inspirés de racisme et de xénophobie. C'est à ce moment-là que le CRAN intervient volontairement?.

Le 16 décembre 2010, le Procureur du Roi rend un avis sur la procédure pénale et conclut qu'il n'y a pas lieu d'interdire la publication de l'ouvrage. Pour motif de connexité, les deux affaires seront jointes par le jugement du 21 juin $2010^{10}$.

Un jugement du 4 avril 2011 reconnait que les demandes de Monsieur Mbutu Mondondo sont recevables. Par ailleurs, le tribunal a reconnu sa compétence matérielle pour statuer sur le fond de ce litige.

Le 10 février 2012, le Tribunal de première instance de Bruxelles déclare les demandes de Monsieur Mbutu Mondondo et du CRAN non fondées et les déboute. II déclare également les demandes de la

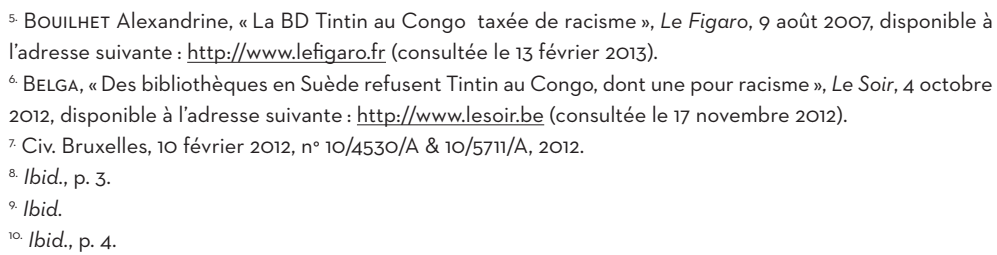


SA Moulinsart et des éditions Casterman non fondées et les déboute".

Rappelons que la SA Moulinsart et les Éditions Casterman mettaient l'accent sur la prescription de l'action principale et réclamaient pour chacun une indemnité de 15.000 euros pour procédure téméraire et vexatoire à titre subsidiaire ${ }^{12}$. La SA Moulinsart à titre subsidiaire se prévalait de l'article 25 de la Constitution afin de rendre l'action principale non recevable.

À titre principal, la partie demanderesse voulait notamment faire cesser la vente des différentes éditions de l'album. Elle souhaitait également que le jugement soit publié dans différents journaux (aux frais de la SA Moulinsart et des éditions Casterman) ainsi que sur le site internet «http:www.tintin.com ». A titre subsidiaire, le CRAN et Monsieur Mbutu Mondondo demandaient notamment d'insérer pour les éditions à venir un avertissement contre le caractère offensant à l'égard des personnes noires et le contexte colonial dans lequel cette bande dessinée a été réalisée ou d'insérer un bandeau et une préface comme cela est pratiqué au Royaume-Uni avec l'édition Egmont.

Insatisfaits de la décision du Tribunal de première instance de Bruxelles, le CRAN et Monsieur Mbutu Mondondo ont interjeté appel'13. La Cour d'appel de Bruxelles a déclaré la demande non fondée et s'exprimera en ces mots:

" S'il fallait suivre les appelants, pour lesquels il suffirait de prendre en considération la simple intention de publier un ouvrage, il faudrait alors interdire aujourd'hui, par exemple, la publication de certaines œuvres de Voltaire, dont le racisme, notamment à l'égard des noirs et des juifs, était inhérent à sa pensée, ainsi que des pans entiers de la littérature, ce qui ne peut être admis, dès lors que l'écoulement du temps doit être pris en compte. Hergé s'est borné à réaliser une œuvre de fiction dans le seul but de divertir ses lecteurs. Il y pratique un humour candide et gentil. Il s'agit avant tout d'un témoignage de l'histoire commune de la Belgique et du Congo à une époque donnée $»^{14}$. 
Mémoire et Politique

\section{Enjeux politiques liant l'affiaire « Tintin au Congo »}

\section{Un uélément moral» comme garantie de la liberté d'expression}

En Belgique, une loi permet de réprimer certains actes inspirés par le racisme ou la xénophobie. II s'agit de la loi du 30 juillet $1981^{15}$. Toutefois, cette loi ne réprime pas l'opinion raciste mais bien l'incitation à la haine ${ }^{16}$. Elle punit des déclarations faites en public qui incitent à la discrimination et à la haine ${ }^{17}$. Dans la décision du tribunal de première instance de Bruxelles, le 10 février 2012, la partie demanderesse base son action sur cette loi et invoque une série d'articles. Trois articles nous intéressent particulièrement, il s'agit des articles $20.3^{\circ}, 20.4^{\circ}$ et 21.

L'article $20.3^{\circ}$ prévoit de l'incitation à la discrimination ou la ségrégation. L'article $20.4^{\circ}$ évoque de l'incitation à la haine ou la violence. Enfin l'article 21 traite des idées fondées sur la supériorité ou la haine raciale ${ }^{18}$.

Pour qu'il y ait une incitation et une diffusion d'idées fondées sur la supériorité ou la haine raciale, le Tribunal explique qu'un élément moral doit être trouvé. Dans son avis, le Procureur du Roi de Bruxelles souligne la définition de l'élément moral attribuée par la Cour constitutionnelle :

« [...] la volonté d'attiser la haine à l'égard d'un groupe de personnes ou de justifier la mise en place d'une politique discriminatoire ou ségrégationniste $n^{19}$.

Il semble qu'Hergé n'ait jamais eu cette intention d'inciter à la haine raciale ; cet argument est partagé aussi bien par Le Procureur du Roi que par le tribunal. L'élément moral joue donc un rôle très important pour garantir la liberté d'expression ${ }^{20}$.

15. L. du 30 juillet 1981 tendant à réprimer certains actes inspirés par le racisme ou la xénophobie, M.B., 8 août 1981.

16. JAMIN Jérôme, «Radicalisme et extrémisme de droite ", in Bounon Frédéric et Reuchamps Min (dir.), Les systèmes électoraux de la Belgique, Bruxelles, Bruylant, 2012, p. 226.

17. Jamin Jérôme, "Belgique: Trente ans de lutte contre le racisme, bilan et perspectives ", La revue nouvelle, $n^{\circ} 4$, avril 2013 , p. 76.

18. Civ. Bruxelles, 10 février 2012, nº 10/4530/A \& 10/5711/A, 2012, p. 14

19. Couvreur Daniel, «Interdire Tintin au Congo serait inconstitutionnel », Le Soir, 28 octobre 2011, disponible

20. Couvreur Daniel, op. cit. 


\title{
Un oubli qui serait la conséquence d’une concurrence mémorielle
}

La façon dont Hergé représente la nation congolaise et ses habitants constitue une mémoire collective des Belges et des idées paternalistes durant les années 1930. On peut parler de mémoire collective ${ }^{21}$ car c'est de cette façon que les citoyens belges se représentaient le peuple congolais et les Belges colonisateurs. Hergé en a témoigné :

\begin{abstract}
" J'étais nourri des préjugés du milieu dans lequel je vivais. C'était en 1930, je ne connaissais de ce pays que ce que les gens en racontaient à l'époque [...]. Je les ai dessinés, ces Africains, dans le pur esprit paternaliste qui était celui de l'époque en Belgique. Si j'avais à le refaire, je le referais tout autrement, c'est sûr ${ }^{22}$.
\end{abstract}

Nous pensons que dans cette affaire nous sommes face à une concurrence mémorielle ${ }^{23}$ où une mémoire collective relayée par Monsieur Mbutu Mondondo avait l'intention de prendre le dessus sur une autre relayée par Hergé et de dénoncer de manière indirecte les oublis de cette dernière. À cet égard, I'historien français, JeanClément Martin va apporter une information intéressante sur la notion d'oubli et de concurrence mémorielle. II dira que :

"Les rivalités mémorielles, [...], ont pour but immédiat de faire ressortir ce qui a été oublié par la Mémoire d'une communauté... $n^{24}$.

Le célèbre philosophe français, Paul Ricœur nous éclaire sur cette notion d'oubli qui entre en lien avec la mémoire et l'histoire. Il explique que l'oubli a trait à la notion de trace et que ces deux éléments ont en commun la notion d'effacement et de destruction ${ }^{25}$. Cette dynamique concurrentielle ne pourrait-elle aboutir, dans le cas d'une prédominance d'une mémoire collective sur une autre, à un oubli des représentations et perceptions des Belges durant les années 1930 ?

\section{Vers une reconnaissance des torts commis aul congo belge}

Un article de l'historien, Bogumil Jewsiewicki traite de la réparation due à la colonisation et l'esclavage des pays occidentaux en Afrique. II

\footnotetext{
21. Voy. la décision de la Cour d'Appel qui fait référence à «l'histoire commune de la Belgique et du Congo ", voy. BeLGA, «Tintin au Congo n'est pas raciste, conclut la Cour d'appel », op.cit.

22. BELGA, "Tintin au Congo n'est pas raciste ", Le Soir, 10 février 2012, disponible à l'adresse suivante: http:// www.lesoir.be (consultée le 17 novembre 2012).

23. La concurrence mémorielle renvoie «à la compétition complexe et parfois douloureuse entre des groupes sociaux (entre eux ou vis-à-vis d'une autorité) pour défendre et promouvoir le souvenir de certains faits historique ». GRANDJEAN Geoffrey et JAMIN Jérôme, La concurrence mémorielle, Paris, Armand Colin, coll. "Recherches », 2011, p. 13.

24. MARTIN Jean-Clément, "Histoire, mémoire et oubli pour un autre régime d'historicité ", Revue d'histoire moderne et contemporaine, $n^{\circ} 47-4$, avril 2000, p. 798.

25. RicoeUr Paul, « Mémoire, Histoire, Oubli », Esprit, mars 2006, p. 27.
} 
explique que le présent est aujourd'hui saisi pour redresser des injustices dans le passé ${ }^{26}$. Il redonnera une définition d'un philosophe ghanéen Anthony Appiah :

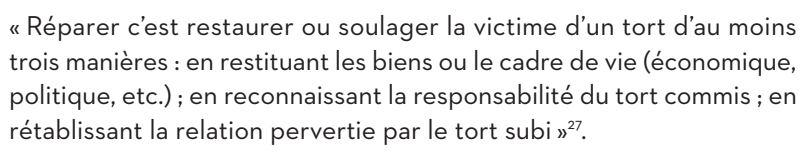

Nous souhaitons ici nous focaliser sur une reconnaissance de responsabilité du tort commis. La Belgique aujourd'hui n'a pas adopté de loi reconnaissant les torts commis à l'égard du Congo belge durant les années 1930.

Selon nous, cette affaire judiciaire peut relancer le débat vers une reconnaissance de ces atrocités commises à cette époque et sur la possibilité d'avoir une intervention politique. Dans un tel contexte, une institution belge pourrait-elle prendre ses responsabilités, "se mouiller " pour accorder cette reconnaissance?

\section{Enjeux mémoriels liés à l'interdiction de l'ouvrage}

\section{Le concept d'enjeu mémoriel}

Avant d'analyser les différents " enjeux mémoriels ", il nous semble opportun de définir ce concept. Dans le cadre de cette contribution, un enjeu mémoriel est une conséquence possible d'une interdiction d'un ouvrage pour la mémoire collective d'une société. Nous en expliquons trois à la suite de ce point.

\section{Une négaation de la représentation coloniale du passé}

Une première conséquence que pourrait apporter l'interdiction du livre serait à notre sens une négation de la représentation du passé. Or notre société démocratique ne fonctionnerait-elle pas mieux si plutôt que de nier les faits, nous entrions dans le conflit d'idées?

Le philosophe français, Claude Lefort met justement en lien la démocratie et le conflit. Pour ce dernier, il faut voir la démocratie comme « ce milieu de conflits, un milieu dans lequel il faut savoir 
contester ${ }^{28}$.

Nier la manière dont étaient représentés le peuple congolais et les Belges colonisateurs serait à notre sens un frein à une reconnaissance des atrocités commises au Congo.

\section{Un avis qui n'est pas partagé par le gouvernement congolais}

La ministre de la Culture et des Arts en République Démocratique du Congo, Jeannette Kavira Mapera, a qualifié la bande dessinée de "chef-d'œuvre " lors de l'inauguration du premier festival de la bande dessinée qui se tient à Kinshasa. Elle s'exprimera en ces mots:
"Pour le gouvernement congolais, Tintin au Congo est un chef- d'œuvre. Cet album ne blesse en rien la culture congolaise. Aux temps anciens, lorsque ce livre a été écrit et que son créateur a été inspiré, effectivement, les Congolais ne savaient pas parler français. Jusqu'aujourd'hui, le Congolais n'est pas celui qui parle le mieux fran- çais. À cette époque-là, décrite dans l'ouvrage, effectivement, pour remettre le Congolais au travail ou l'inciter à travailler, il fallait utiliser le bâton. [...] Nous avons estimé que c'était un procès intéressé qui n'engage pas le gouvernement congolais ${ }^{29}$.

Ce qu'avance la Ministre à l'occasion du festival est intéressant. En effet, cette dernière appuie à la suite du qualificatif « chef-d'œuvre " une série de remarques sur la manière d'être du congolais à cette époque. Toutefois il serait intéressant de questionner les raisons poussant la Ministre à faire une telle déclaration.

\section{Une décision qui pourrait laire jurisprudence}

Avec une interdiction de "Tintin au Congo ", d'autres œuvres relatant des faits passés ou une pensée passée pourrait bien tomber sous la menace d'une interdiction. Nous pouvons citer à titre d'exemple "Le Village Aérien " de Jules Verne ${ }^{30}$. Cette histoire raconte l'aventure au Congo de deux amis explorateurs. Ils sont accompagnés d'un petit enfant indigène et d'un guide camerounais. Ils y découvriront un peuple inconnu qu'ils étudieront afin de savoir si ce sont véritablement des humains. Dans son livre, Jules Verne va comparer l'intelligence d'un adulte noir à celle d'un enfant blanc de six ans. À l'instar de la

28. LeFORT Claude et RosanvalLon Pierre "À l'épreuve du politique: Dialogue entre Claude Lefort et Pierre Rosanvallon », Esprit, décembre 2011, n¹2, p. 29 .

29. BELGA, "La ministre congolaise de la Culture défend Tintin au Congo ", La Libre Belgique, 21 octobre 2010, disponible à l'adresse suivante : http://www.lalibre.be (consultée le 22 novembre 2012). 30. Verne Jules, Le Village Aérien, Tredition Classics, 2012, 200 p. 
décision rendue par la Cour d'Appel, nous pouvons également citer Voltaire dont certaines de ses œuvres reflètent le racisme, notamment à l'égard des noirs et des juifs ${ }^{31}$.

\section{Conclusion}

Pour conclure, rappelons d'abord que cette œuvre a été écrite à l'époque du colonialisme qui voyait circuler de nombreuses idées paternalistes. La décision de justice sur cette affaire le rappelle d'ailleurs :

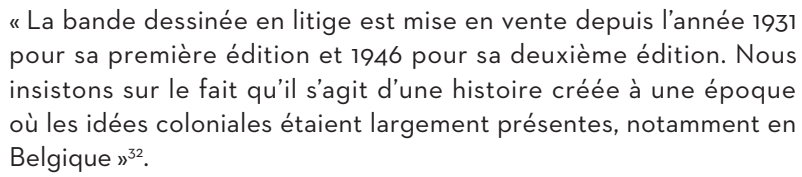

La différence de mentalité entre cette époque et la nôtre est bien présent. Nous ne pensons plus comme nos ancêtres et nous devons tirer des leçons du passé.

"Tintin au Congo " peut par ailleurs constituer un outil pédagogique destiné aux enfants puisqu'elle peut rappeler cette mémoire collective représentant le peuple congolais et les Belges colonisateurs. Bien évidemment, nous ne voulons pas dire que ce n'est que grâce à cette bande dessinée que les enfants apprendront tout du colonialisme. Cette dernière pourrait constituer une introduction aux thèmes de la colonisation et de l'esclavage. Ensuite, l'histoire sur ces mêmes thèmes pourrait être enseignée au travers de livres historiques.

Si dans la bande dessinée le peuple congolais est caricaturé, il ne faut pas perdre de vue que le Belge et le colonisateur le sont également. Hergé représente le Blanc comme quelqu'un se croyant supérieur aux Noirs, criant sur les villageois, les traitant de fainéants. Au final, dans l'ouvrage, les Blancs sont «les mauvais ». Les « blancs " sont caricaturés de la même façon que les «noirs".

Enfin, l'œuvre d'Hergé fait tout autant partie de la culture belge que congolaise. De plus, la Cour d'Appel parle de "témoignage de l'histoire commune de la Belgique et du Congo "13. Dans cette perspective, la mémoire collective permet de préserver les représentations passées fondant, d'une façon ou d'une autre, les racines et les

31. BeLGA, «Tintin au Congo n'est pas raciste, conclut la Cour d'appel ", Le Vif L'Express, 5 décembre 2012, disponible à l'adresse suivante $:$ http://focus.levif.be, (consultée le 5 décembre 2012). 32. Civ. Bruxelles, 10 février 2012, n 10/4530/A \& 10/5711/A, 2012, p. 13.

33. BELGA, «Tintin au Congo n'est pas raciste, conclut la Cour d'appel », Le Vif L'Express, op. cit. 
Mémoire et Politique

01

repères d'une société.

Peut-on analyser au regard de nos valeurs actuelles véhiculées en Belgique (comme par exemple l'égalité de traitement) cette bande dessinée empreinte d'un paternalisme et d'idées coloniales tels qu'ils existaient à l'époque ou, au contraire, doit-on analyser cette dernière au regard du contexte de l'époque, à partir d'un « imaginaire colonial »?

Le concept d'imaginaire est expliqué par le philosophe Cornelius Castoriadis. Selon ce dernier, il ne faut pas séparer l'imaginaire de la réalité ${ }^{34}$. En effet, l'imaginaire a cette capacité à produire des valeurs, des normes et des vérités orientant nos comportements ${ }^{35}$.

L'imaginaire colonial caractérise donc ces valeurs, ces normes et ces vérités qu'un peuple, un groupe, une ethnie avait à une époque bien déterminée, à savoir celle du colonialisme. L'imaginaire colonial nous permet de nous conscientiser sur la vision des peuples à cette époque, vision qui évolue avec le temps et qui oriente nos comportements. 